\title{
A Rapid Transfer Alignment Method for SINS Based on the Added Backward-Forward SINS Resolution and Data Fusion
}

\author{
Xixiang Liu, ${ }^{1,2}$ Xiaosu Xu, ${ }^{1,2}$ Yiting Liu, ${ }^{1,2}$ and Lihui Wang ${ }^{1,2}$ \\ ${ }^{1}$ School of Instrument Science \& Engineering, Southeast University, Nanjing 210096, China \\ ${ }^{2}$ Key Laboratory of Micro-Inertial Instrument and Advanced Navigation Technology, Ministry of Education, Nanjing 210096, China
}

Correspondence should be addressed to Xixiang Liu; scliuseu@163.com

Received 28 January 2013; Accepted 25 June 2013

Academic Editor: Joao B. R. Do Val

Copyright (c) 2013 Xixiang Liu et al. This is an open access article distributed under the Creative Commons Attribution License, which permits unrestricted use, distribution, and reproduction in any medium, provided the original work is properly cited.

\begin{abstract}
Two viewpoints are given: (1) initial alignment of strapdown inertial navigation system (SINS) can be fulfilled with a set of inertial sensor data; (2) estimation time for sensor errors can be shortened by repeated data fusion on the added backward-forward SINS resolution results and the external reference data. Based on the above viewpoints, aiming to estimate gyro bias in a shortened time, a rapid transfer alignment method, without any changes for Kalman filter, is introduced. In this method, inertial sensor data and reference data in one reference data update cycle are stored, and one backward and one forward SINS resolutions are executed. Meanwhile, data fusion is executed when the corresponding resolution ends. With the added backward-forward SINS resolution, in the above mentioned update cycle, the estimating operations for gyro bias are added twice, and the estimation time for it is shortened. In the ship swinging condition, with the "velocity plus yaw" matching, the effectiveness of this method is proved by the simulation.
\end{abstract}

\section{Introduction}

Transfer alignment is a rapid and effective initial alignment method, which is widely used for inertial navigation systems (INSs) in ships and planes. For the quick response requirement of weapon systems, rapidity is always the main aim of the initial alignment. In 1989, a fast transfer alignment method was presented by Kain and Cloutier, in which alignment can be fulfilled in $10 \mathrm{~s}$, and $1 \mathrm{mrad}$ accuracy can be got with swinging movement and "velocity plus attitude" matching [1]. Based on reference 1, a prefilter was added before the Kalman filter by Spalding [2]. Together with the former, the calculation amount was reduced and alignment time was shortened. And in this method, 1 mrad accuracy was reached within $6 \mathrm{~s}$, when the update frequency of reference data was $1 \mathrm{~Hz}$. Aviation transfer alignment experiments on Apache helicopter and F-16 fighter were conducted by Shortelle and Graham, respectively [3-5], and the test results indicated that, when the update frequency was $12.5 \mathrm{~Hz}$, the alignment time could be reduced to $5 \mathrm{~s}$ and the accuracy could reach $1 \mathrm{mrad}$, with "velocity plus attitude" matching and Wing-Rock tactical action.
In the above references, the rapidity of initial alignment was studied, while the estimation for inertial sensor errors was not taken into account or not given sufficient attention. To those strapdown INSs, which are composed of low or medium accuracy initial sensors, sensor errors will decrease navigation accuracy greatly, due to the great drift of gyros after the long time storage [6]. So, it is certain that navigation accuracy will be improved if the sensor errors are estimated in the transfer alignment process. But the observability degree of each state variable in a Kalman filter for transfer alignment relies on the information matching method and the motion of carrier $[7,8]$. With swinging movement and "velocity plus attitude" matching, an effective estimation for sensor errors cannot be completed within $10 \mathrm{~s}$ for it usually lasts for several minutes [9].

In this paper, after transfer alignment process in ship between the master INS (MINS) and slave INS has been studied, a faster transfer alignment method is introduced, in which the estimation for gyro bias can be fulfilled in a shorter time. In ship, the accuracy of platform INS (PINS) is usually several magnitudes higher than that of strapdown INS. The PINS is used as MINS while the strapdown INS 
is used as slave INS, and the navigation parameters from MINS are used as reference data. In the paper, the slave INS and strapdown INS are both abbreviated as SINS. In the integration of M/S INS, flexure deformation is a key factor which will determine transfer alignment accuracy [10]. In this paper, "velocity plus yaw" matching method is chosen due to the fact that the "attitude" matching method is easily suffered by flexure deformation [11, 12], while "velocity" matching method can ensure the rapidity and accuracy of estimation for horizontal misalignment angles [8]. According to the analysis based on piece-wise constant systems (PWCS) linear theory [7], with the excitation of the wave swinging, the velocity errors, misalignment angles, and gyro bias are the observable parameters [13], but, unfortunately, the estimation for gyro bias is a time-consuming process which usually lasts for several minutes.

To deal with the contradiction between the rapidity of alignment and time consumption of gyro bias estimation, two viewpoints are put forward by analyzing the alignment process of SINS. Firstly, different from that in PINS, the direct corresponding relationship between inertial senor data and misalignment angles in SINS is severed by the mathematical platform. Thus, we can adjust the calculated mathematical platform $n^{\prime}$ constantly to speed up the initial alignment by the repeated SINS resolution with the same set of sensor data. Secondly, analysis indicates that, with a Kalman filter, a new estimation for state vector will be provided. With closeloop correction, new initial navigation parameters and new compensation parameters for gyro bias will be produced for the next navigation period, and with the above new ones, new navigation results will be generated even built on the same set of sensor data. So with the repeatedly data fusion of the added backward-forward SINS resolution results and the external reference data, the estimation time for sensor error will be shortened. With the above two viewpoints, a rapid transfer alignment method based on the added backward-forward SINS resolution, without any changes to the Kalman filter, is designed in detail. In one reference data update cycle, with a normal resolution and data fusion, an added backward and an added forward resolutions, and their data fusions, the time consumed for gyro bias estimation is effectively shortened. The effectiveness of this method is proved by simulation results.

This thesis is constructed as follows. In Section 2, the transfer alignment model based on "velocity plus yaw" matching is built, and the reasons why the observability degree of gyro bias is low are analyzed. In Section 3, after the alignment process of INS is studied and compared, a possible way to increase SINS alignment speed is introduced, and the backward-forward SINS resolution is designed. In Section 4, the transfer alignment model based on the added backward-forward resolution is also designed in detail. And in Section 5, the effectiveness of this method is proved by simulation. Finally, some conclusions are given.

\section{Transfer Alignment Model Based on "Velocity Plus Yaw" Matching Method}

2.1. System Equation. Choose velocity errors, misalignment angles, and gyro bias as the state vector of the system,

$$
\mathbf{X}=\left[\begin{array}{llllllll}
\delta V_{E} & \delta V_{N} & \phi_{E} & \phi_{N} & \phi_{U} & \varepsilon_{x} & \varepsilon_{y} & \varepsilon_{z}
\end{array}\right]^{T},
$$

where $\delta V_{E}$ and $\delta V_{N}$ are east and north velocity errors, respectively, $\phi_{E}, \phi_{N}$, and $\phi_{U}$ are misalignment angles of pitch, roll, and yaw, respectively, $\varepsilon_{x}, \varepsilon_{y}$, and $\varepsilon_{z}$ are gyro bias along $x, y$, and $z$ axes. To ships which sail on the sea, the height and upward velocity can be set as zero.

The system state equation can be constructed as

$$
\dot{\mathbf{X}}(t)=\mathbf{A}(t) \mathbf{X}(t)+\mathbf{W}(t),
$$

where $\mathbf{A}(t)$ is the state matrix and $\mathbf{W}(t)$ is the system noise matrix. With the system vector, velocity error, and misalignment angle equations, the state matrix $\mathbf{A}(t)$ can be expressed as

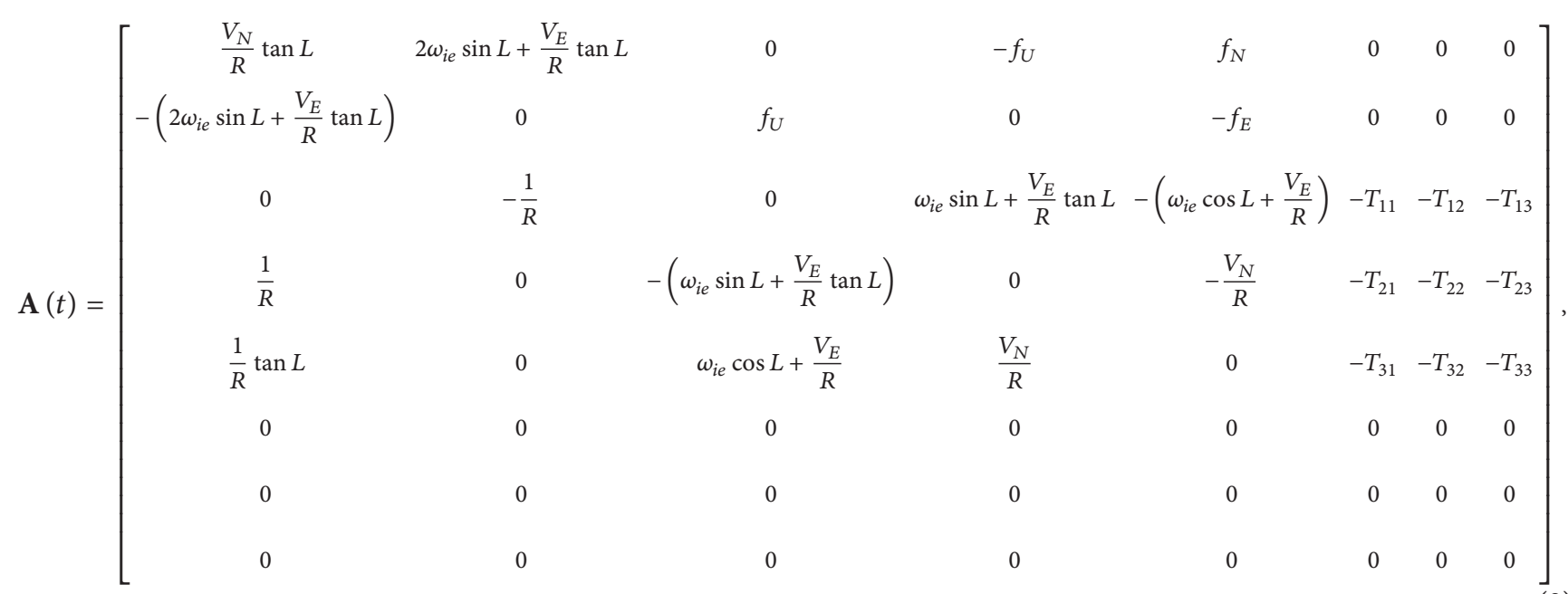




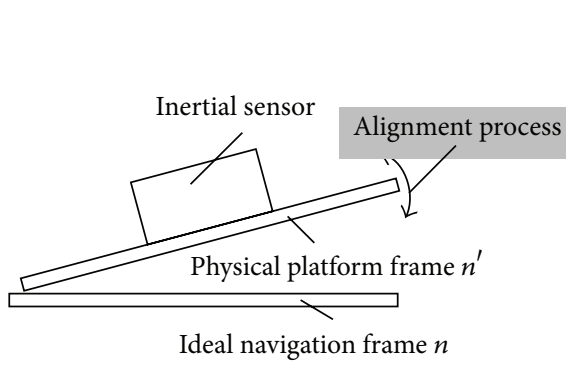

(a) PINS

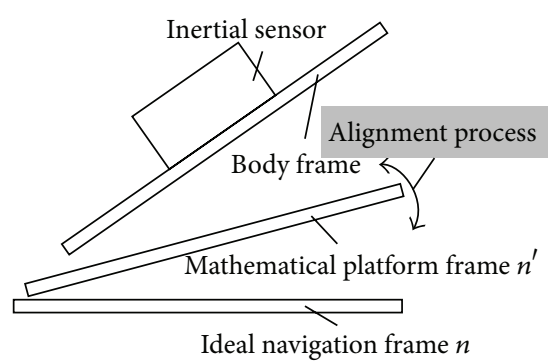

(b) SINS

FIGURE 1: Alignment process for INS.

where $V_{E}$ and $V_{N}$ are the east and north velocity respectively, $\omega_{i e}$ and $R$ are the rotational angular rate and radius of the Earth, $L$ is the latitude of ship position, $f_{E}, f_{N}$, and $f_{U}$ denote the projection of the accelerometer measured data $\mathbf{f}^{b}$ in navigation frame $n$, and $T_{i j}(i, j=1,2,3)$ are the elements of the direct cosine matrix (DCM) of MINS.

2.2. Measurement Equation. The differences of velocity and yaw between MINS and SINS are used as measurement data for data fusion. Then, the measurement vector is as

$$
\mathbf{Z}=\left[\begin{array}{lll}
V_{E}-V_{M E} & V_{N}-V_{M N} & Y_{E}-Y_{M E}
\end{array}\right]^{T},
$$

where $V_{E}, V_{N}$ and $Y_{E}$ are the east, north velocity, and yaw from SINS respectively, $V_{M E}, V_{M N}$, and $Y_{M E}$ are from MINS, respectively.

The measurement equation can be constructed as

$$
\mathbf{Z}(t)=\mathbf{H}(t) \mathbf{X}(t)+\mathbf{V}(t)
$$

where $\mathbf{H}(t)$ is measurement matrix and $\mathbf{V}(t)$ is measurement noise matrix. According to the relationship between measurement and state vectors, the measurement matrix $\mathbf{H}(t)$ can be expressed as

$$
\mathbf{H}=\left[\begin{array}{cccccc}
1 & 0 & 0 & 0 & 0 & \\
0 & 1 & 0 & 0 & 0 & 0_{3 \times 3} \\
0 & 0 & -\frac{T_{12} T_{32}}{T_{12}^{2}+T_{22}^{2}} & -\frac{T_{22} T_{32}}{T_{12}^{2}+T_{22}^{2}} & &
\end{array}\right] .
$$

2.3. Observability Degree of Each State Variable. After the analysis of the model combined with (2) and (5) by PWCS theory, we can conclude that all of the eight selected variables are observable, but the observability degrees are different from each other [13]. The simulation results in Section 5.2 indicate that the horizontal velocity errors and yaw misalignment converge immediately, that the estimation for horizontal misalignment angles lasts for 10 20 s, and that the estimation for gyro bias lasts for $3 \sim 5 \mathrm{~min}$, which means that the degrees of velocity errors and yaw misalignment are the highest, those of horizontal misalignment angles are moderate, and those of gyro bias are the lowest.

The reasons which cause the different degrees of errors are as follows. The gyro bias needs integration to be reflected on misalignment angles, and the horizontal misalignment angles need projection reflected on acceleration. Further integration of acceleration, gyro bias can be reflected on velocity errors. In this process, from gyro bias to velocity errors, integrating operations are needed twice, and from horizontal misalignment angles to velocity errors, only one is needed. When velocity errors and yaw misalignment are selected as components of a measurement vector, there is no doubt that observability degrees of gyro bias are lowest, while those of velocity errors and yaw misalignment are highest, which are determined by the mechanism of transfer alignment. To shorten the estimation time for gyro bias, some other novel ways should be sought.

\section{A New Way to Speed Up SINS Alignment}

3.1. Analysis of Alignment Process in INS. An accepted viewpoint is that in navigation sensor data is of real-time significance. Based on initial navigation parameters, INS obtains the attitude, velocity, and position by the integration of sensor data. After integration, the sensor data can be discarded.

Take the alignment process of PINS as an example. As shown in Figure 1(a), $n^{\prime}$ frame denotes the physical platform and $n$ frame denotes the ideal navigation frame. In PINS, inertial sensors are installed on the physical platform. Inertial sensors measure the ship motion in $n^{\prime}$ frame, and navigation resolution is also executed in this frame; however, reference data are from $n$ frame. Before aligning, misalignment angles between $n^{\prime}$ and $n$ frames will cause the differences between navigation resolution values in $n^{\prime}$ frame and reference values in $n$ frame. Because these differences can reflect the misalignments, transfer alignment can be finished with the model in Section 2. In the alignment process, with the above differences caused by misalignment angles, physical platform can be adjusted to coincide with the ideal frame. In other words, in PINS, sensor data reflect the magnitude of misalignment angle and other errors, and there is direct 
relationship between sensor data and misalignment angles, so the sensor data are of real-time significance. And the adjustment for physical platform to get new sensor data is a time-consuming process.

At the same time, in SINS, the alignment method is derived from that of PINS, and so, the alignment must be a time-consuming process for the need of real-time sensor data and the adjustment of platform. But in INS, as far as the process of alignment is concerned, maybe, sensor data is of real-time significance for PINS, but not for SINS.

In SINS, as shown in Figure 1(b), a mathematical platform replaces the physical platform in PINS and inertial sensors are directly installed on the ship. Here, the ship body is named $b$ frame and the mathematical platform is named $n^{\prime}$ frame. In alignment, the adjusting process of the $n^{\prime}$ frame is the same with that in PINS, while, the difference is that the calculated sensor data in $n^{\prime}$ frame is the projection of measured sensor data from $b$ frame. Only the projection data in $n^{\prime}$ frame reflect the misalignment angles between $n^{\prime}$ and $n$ frames, but sensor data cannot. The calculated and measured sensor data are connected by calculated DCM $\mathrm{C}_{b}^{n^{\prime}}$. In comparison with that in PINS, the direct relationship between sensor data and misalignment angles is severed by the mathematical platform. Then, the $n^{\prime}$ frame can be adjusted constantly by the repeated calculation for DCM $\mathbf{C}_{b}^{n^{\prime}}$ with a same set of sensor data, which means that alignment time can be shortened without too much time to be spent in sampling real-time sensor data.

The above set of data can be seen as that, when the ship keeps an ideal static state and there are no sensor errors, all measured sensor data are equal and can be dealt with as a series of single data. In engineering, because of the errors from sensor and reference data, the update frequency of reference data, and so forth, a set of sensor data and reference data should be used for repeated SINS resolution (added backward-forward resolution) and repeated data fusion to improve the alignment accuracy.

Also, different from PINS, SINS is a digital system, in which there is only numerical data, no mass, spring, and resistance. So the adjustment for mathematical platform can be as fast as lightning.

3.2. Backward-Forward SINS Resolution. The above analysis indicates that the alignment of SINS can be fulfilled with a set of same data, which brings a new problem-the way to use these data.

In SINS, with initial attitude, velocity, and position, the navigation resolution is the real-time updating process for navigation parameters with sensor data by integrated calculation. In this process, ship moves from the origin to the end. In backward-forward SINS resolution, as shown in Figure 2, backward SINS resolution is the process in which ship moves from the end to the origin-a reverse process of normal navigation, and forward resolution is that from the origin to the end-a repeated process of normal navigation. From the basic SINS resolution algorithm, the deduction of backward-forward SINS algorithm is shown as follows.

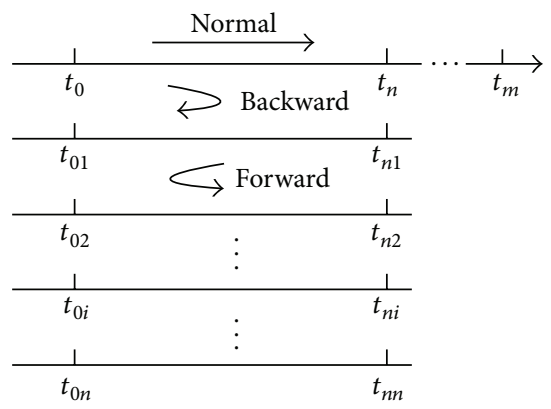

FIGURE 2: The process of backward-forward SINS resolution.

3.2.1. Normal Resolution Algorithm in SINS. The navigation resolution algorithm is [14]

$$
\begin{gathered}
\dot{\mathbf{C}}_{b}^{n}=\mathbf{C}_{b}^{n}\left(\boldsymbol{\omega}_{n b}^{b} \times\right), \\
\dot{\mathbf{V}}^{n}=\mathbf{C}_{b}^{n} \mathbf{f}^{b}-\left(2 \boldsymbol{\omega}_{i e}^{n}+\boldsymbol{\omega}_{e n}^{n}\right) \times \mathbf{V}^{n}+\mathbf{g}^{n}, \\
\dot{L}=\frac{V_{N}^{n}}{R}, \quad \dot{\lambda}=\frac{V_{E}^{n} \sec L}{R},
\end{gathered}
$$

where $\boldsymbol{\omega}_{n b}^{b}=\boldsymbol{\omega}_{i b}^{b}-\left(\mathbf{C}_{b}^{n}\right)^{T}\left(\boldsymbol{\omega}_{i e}^{n}+\boldsymbol{\omega}_{e n}^{n}\right), \boldsymbol{\omega}_{e n}^{n}=$ $\left[-V_{N}^{n} / R V_{E}^{n} / R\left(V_{E}^{n} \tan L\right) / R\right]^{T}, \mathbf{A}_{B C}^{D}\left(\right.$ such as $\left.\boldsymbol{\omega}_{i e}^{n}\right)$ denotes the projection of a motion vector $\mathbf{A}$ which means the relative motion from $C$ frame to $B$ frame in $D$ frame, $C_{b}^{n}$ is the DCM, $\mathbf{V}^{n}=\left[\begin{array}{lll}V_{E}^{n} & V_{N}^{n} & V_{U}^{n}\end{array}\right]$ is the velocity vector, $L$ and $\lambda$ are the latitude and longitude, respectively, and $(\cdot \times)$ denotes the antisymmetric matrix of the vector “.”.

For the recursive update with computer, formulas (7) must be translated into discrete form in a certain sampling cycle and navigation resolution update cycle. For a ship, which always undergoes a low dynamical movement, a single sampling resolution algorithm can be adopted, which means only one sample of sensor data per navigation cycle [15-17]. Supposing the above cycle times are both equal to $T_{s}$, then the discrete form can be expressed as follows [14]

$$
\begin{gathered}
\mathbf{C}_{b k}^{n}=\mathbf{C}_{b k-1}^{n}\left(\mathbf{I}+T_{s} \boldsymbol{\omega}_{n b k}^{b} \times\right), \\
\mathbf{V}_{k}^{n}=\mathbf{V}_{k-1}^{n}+T_{s}\left[\mathbf{C}_{b k-1}^{n} \mathbf{f}_{k-1}^{b}-\left(2 \boldsymbol{\omega}_{i e k-1}^{n}+\boldsymbol{\omega}_{e n k-1}^{n}\right) \times \mathbf{V}_{k-1}^{n}+\mathbf{g}^{n}\right], \\
L_{k}=L_{k-1}+\frac{T_{s} V_{N k-1}}{R_{M}+h_{k-1}}, \quad \lambda_{k}=\lambda_{k-1}+\frac{T_{s} V_{E k-1} \sec L_{k-1}}{R_{N}+h_{k-1}},
\end{gathered}
$$

where $k$ denotes recursive number. Without consideration of calculation error, formulas (8) are composed of the basic update equations for navigation resolution.

3.2.2. Backward Resolution Algorithm in SINS. As shown in Figure 2, in a backward resolution process, a ship needs to return from the end to the origin. So in the normal process, 
all sensor data must be stored. According to the formulas (8), the resolution process can be expressed as follows:

$$
\begin{gathered}
\mathbf{C}_{b k-1}^{n}=\mathbf{C}_{b k}^{n}\left(\mathbf{I}+T_{s} \boldsymbol{\omega}_{n b k}^{b} \times\right)^{-1} \\
\approx \mathbf{C}_{b k}^{n}\left(\mathbf{I}-T_{s} \boldsymbol{\omega}_{n b k}^{b} \times\right) \approx \mathbf{C}_{b k}^{n}\left(\mathbf{I}-T_{s} \boldsymbol{\omega}_{n b k-1}^{b} \times\right) \\
\mathbf{V}_{k-1}^{n}=\mathbf{V}_{k}^{n}-T_{s}\left[\mathbf{C}_{b k-1}^{n} \mathbf{f}_{k-1}^{b}-\left(2 \boldsymbol{\omega}_{i e k-1}^{n}+\boldsymbol{\omega}_{e n k-1}^{n}\right) \times \mathbf{V}_{k-1}^{n}+\mathbf{g}^{n}\right] \\
\approx \mathbf{V}_{k}^{n}-T_{s}\left[\mathbf{C}_{b k}^{n} \mathbf{f}_{k}^{b}-\left(2 \boldsymbol{\omega}_{i e k}^{n}+\boldsymbol{\omega}_{e n k}^{n}\right) \times \mathbf{V}_{k}^{n}+\mathbf{g}^{n}\right] \\
L_{k-1}=L_{k}-\frac{T_{s} V_{N k-1}}{R} \approx L_{k}-\frac{T_{s} V_{N k}}{R}, \\
\lambda_{k-1}=\lambda_{k}-\frac{T_{s} V_{E k-1} \sec L_{k-1}}{R} \approx \lambda_{k}-\frac{T_{s} V_{E k} \sec L_{k}}{R},
\end{gathered}
$$

where $k$ is reduced from $n$ to 0 . As shown in Figure $2, t_{n 1}$ is both the starting point in the period $t_{n 1} \sim t_{01}$ and the ending point in the period $t_{0} \sim t_{n}$. Take the attitude, velocity, and position at $t_{n}$ as the initial attitude velocity and position at $t_{n 1}$, and the backward resolution can be fully realized. In the period of $t_{0} \sim t_{n}$ and $t_{n 1} \sim t_{01}$, with the same recursive number $k$, the ship maintains the same attitude, velocity, and position and maintains the same acceleration but opposite in direction. Some errors are induced by the approximation in formulas (9), and all these errors can be ignored when the resolution cycle is short enough. The simulation in Section 3.2.3 proves that the above approximation is effective.

After the backward resolution, a forward resolution should be made in order for the ship to return from the origin to the end. In the forward resolution, formulas (8) can be used.

3.2.3. Simulation on Backward-Forward SINS Resolution. The sensor errors are listed in Table 1. And we assume that the ship is in a bad-moderate sea condition [18], and the ship swinging parameters are listed in Table 2. Ship initial attitudes are set as 0 , and the ship is assumed without linear motion, and located north latitude $32^{\circ}$ and east longitude $118^{\circ}$. Sensor sampling and navigation resolution cycle $T_{s}$ is set as $10 \mathrm{~ms}$.

Simulation results are shown in Figure 3, in which (a), (b), and (c) show the resolution results of pitch, east velocity, and latitude. The dot and solid lines denote the simulation with no sensor error and with sensor error, respectively. The simulation is divided into three stages: firstly, in the $t_{0} \sim t_{n}$ period, sensor data are measured and stored, normal navigation is resolved, and this period lasts for $1 \mathrm{~s}$; secondly, in the $t_{n 1} \sim t_{01}$ period, backward resolution is run; and finally, in the $t_{02} \sim t_{n 2}$ period, forward resolution is run. In the last two stages, the time consumed is determined by the performance of computer.

In Figure 3, without consideration of calculating the error, the ship can move from the end to the origin and move from origin to end with the backward and forward resolutions, respectively.

But the above processes also indicate that, with only backward or forward or backward-forward SINS resolution, no new information will be generated.
TABLE 1: Sensor errors.

\begin{tabular}{ccccc}
\hline & \multicolumn{2}{c}{ Gyro bias } & \multicolumn{2}{c}{ Acce bias } \\
& Constant & Random & Constant & Random \\
\hline$x$ & $0.5^{\circ} / \mathrm{h}$ & $0.5^{\circ} / \mathrm{h}$ & $500 \mu \mathrm{g}$ & $500 \mu \mathrm{g}$ \\
$y$ & $0.5^{\circ} / \mathrm{h}$ & $0.5^{\circ} / \mathrm{h}$ & $500 \mu \mathrm{g}$ & $500 \mu \mathrm{g}$ \\
$z$ & $0.5^{\circ} / \mathrm{h}$ & $0.5^{\circ} / \mathrm{h}$ & $500 \mu \mathrm{g}$ & $500 \mu \mathrm{g}$ \\
\hline
\end{tabular}

TABLE 2: Swinging parameters.

\begin{tabular}{lccc}
\hline & Pitch & Roll & Yaw \\
\hline Amplitude $\left({ }^{\circ}\right)$ & 9 & 12 & 14 \\
Cycle $(\mathrm{s})$ & 8 & 10 & 6 \\
\hline
\end{tabular}

\section{Rapid Transfer Alignment Based on the Added Resolution and Data Fusion}

The analysis in Section 3.1 indicates that alignment for SINS can be fulfilled with a set of data, and in this section, we combine a Kalman filter with the added backward-forward SINS resolution aiming to shorten the alignment time.

As shown in Figure 4, transfer alignment model based on Kalman filter introduced in Section 2 and a close-loop correction method are used in this rapid transfer alignment algorithm. Close-loop correction means that, after data fusion, the new estimation for velocity errors and misalignment angles will be fed to SINS to revise those corresponding parameters, and new estimation for gyro bias will be set as new compensation value to participate in the following navigation resolutions. In other words, after data fusion, the mathematical platform $\mathbf{C}_{b}^{n^{\prime}}$ will be adjusted. For a ship, the update frequency of the reference data from MINS is lower than that of SINS navigation resolution. $T_{s}$ and $T_{n}$ are set as update cycle of navigation resolution and reference data, respectively. Also, $T_{n}$ is set as the added backward-forward SINS resolution cycle.

Two transfer alignment methods are compared, and the estimation processes of gyro bias are schematically demonstrated in Figure 5, in which (a) and (b) indicate these two methods, respectively, which are all based on the principle as shown in Figure 4. In the first one, the added backwardforward SINS resolution and data fusion are not used, while they are used in the second. In Figure 5, the dot-dashed lines denote the real gyro bias, while the solid line denotes the estimation of gyro bias. With the Kalman filter and matching method introduced in Section 2, the estimation of gyro bias is a slow process which will be convergent towards real gyro bias after a long time.

As shown in Figure 5(a), when the reference data is available, such as at the point $t_{n}$, data fusion is executed, and a new estimate for the state vector will be produced. With the new estimation for misalignment angles and velocity errors, initial navigation parameters will be reset for the next period, such as $t_{n} \sim t_{2 n}$, which means that the mathematical platform $n^{\prime}$ will be adjusted, and the new compensation value for gyro bias will also be reset. In this method, along with time, data fusion is run only once at every reference data update cycle. 


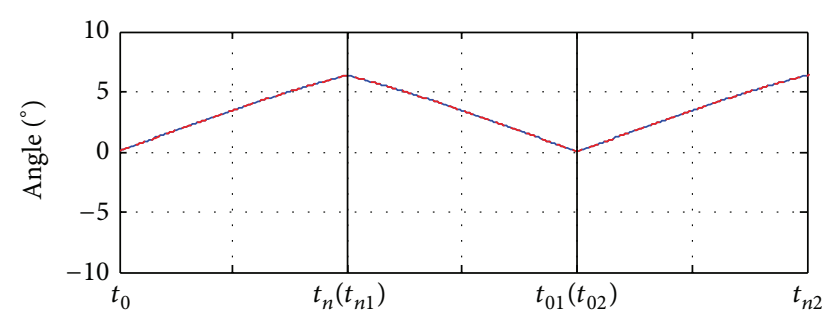

(a) Pitch

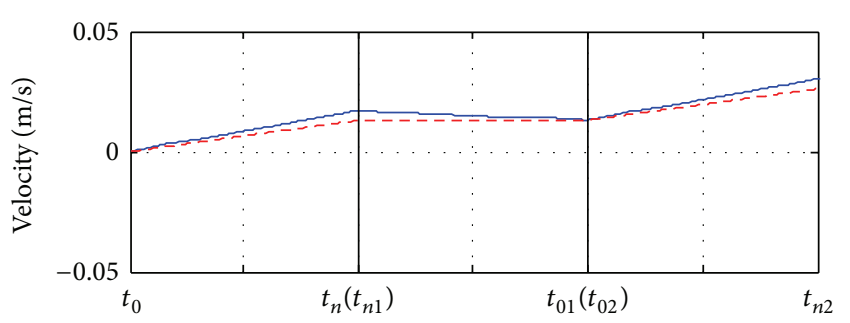

(b) East velocity

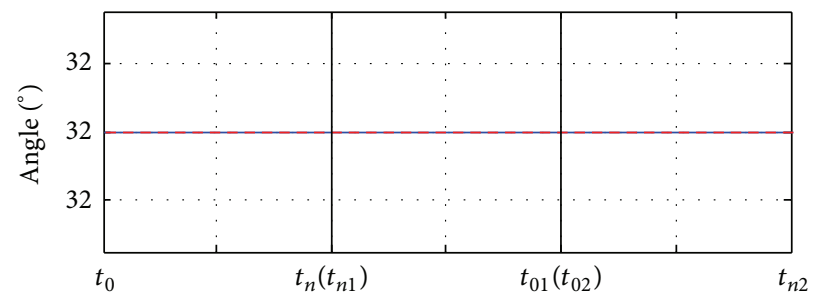

(c) Latitude

FIGURE 3: Simulation of normal and backward-forward SINS resolution.

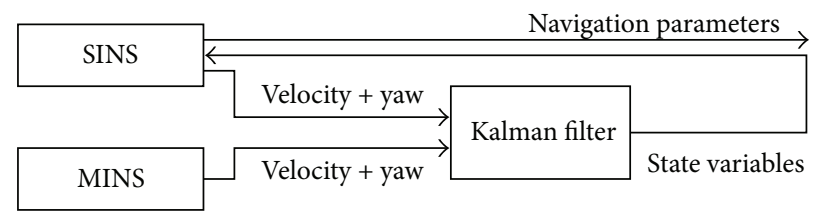

Figure 4: Kalman filter.

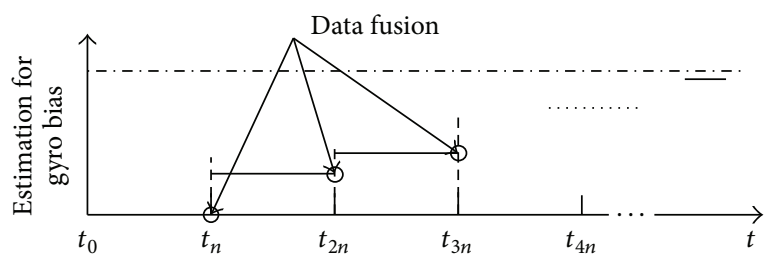

(a) Without added backward-forward SINS resolution and data fusion

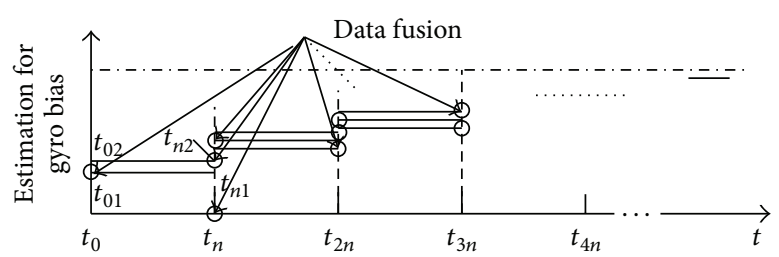

(b) With added backward-forward SINS resolution and data fusion

FIGURE 5: Estimation process for gyro bias.

But, as shown in Figure 5(b), in the same reference data update cycle, such as $t_{0} \sim t_{n}$, at the point $t_{n}$, data fusion will be run, and new estimation will be produced, and so will new initial navigation parameters. And new initial parameters and new compensation value for gyro bias will produce the new navigation parameters at the point $t_{01}$, which are different from those at $t_{0}$. So, a new estimate will be generated at the point $t_{01}$, which is different from that at $t_{0}$, because the measurement vectors for the Kalman filter are different at points $t_{0}$ and $t_{01}$, which is caused by the same reference data but different navigation parameters. Similarly, new information will be got at the point $t_{n 2}$, which is different from that at $t_{n}$. In Figure 5(b), with the added backwardforward SINS resolution and data fusion, the estimating operations for gyro bias and the adjustment for mathematical platform $n^{\prime}$ will be done with two more times. In the second method, even with same observability degrees as in the first one, the estimation time will be shortened, because the estimation frequency is increased.

There is no doubt that the added resolution and data fusion in Figure 5(b) will increase the burden of navigation computer. Though in the last decades, the processing powering of the employed microprocessors has dramatically increased, it is difficult, in some way, to complete a large computation in a relatively short period.

As shown in Figure 6, in this rapid transfer alignment method, three tasks should be completed within one reference data update cycle. (1) Inertial sensor data needs to be sampled and stored and navigation resolution should be run. (2) Reference data needs to be sampled and stored and data fusion should be run. (3) Backward and forward calculations and data fusions should be executed. The first task should be 
executed at every navigation update cycle. The last two tasks should be run in the last navigation update cycle of every reference data update cycle, which means that the above three tasks should be finished in $T_{s}$. Otherwise, the first task in the first navigation cycle of the next reference data cycle will be compromised. It is difficult to finish these three tasks in $T_{s}$ with a computer of limited performance.

However, this problem can be resolved by the full use of resources of high speed computers with the support of real-time multitasking operation system (RTOS), such as VxWorks. In VxWorks, the above three tasks can be set with different priorities. The first can be run preferentially when Task 2 or Task 3 is being run, which means that the first task of the next reference data cycle can be run preferentially when
Tasks 2 and 3 of the previous reference data cycle are being executed. In this method, the idle resources of CPU in the next reference cycle can be used for the tasks 2 and 3 of the previous cycle. The paper is not involved in the programs in RTOS in detail.

\section{Simulation}

5.1. Parameters for Simulation. The ship moving parameters are set as in Section 3.2.3. The ideal velocity and yaw of the ship are used as reference data from MINS after white noise is added. The variance of the white noise is set as $\left[(0.4 \mathrm{~m} / \mathrm{s})^{2}(0.4 \mathrm{~m} / \mathrm{s})^{2}\left(0.3^{\circ}\right)^{2}\right]$. The sensor errors are listed in Table 1 .

The parameters for Kalman filter are

$$
\begin{aligned}
& \mathbf{X}_{0}=\left[\begin{array}{llllllll}
0 & 0 & 0 & 0 & 0 & 0 & 0 & 0
\end{array}\right]^{T},
\end{aligned}
$$

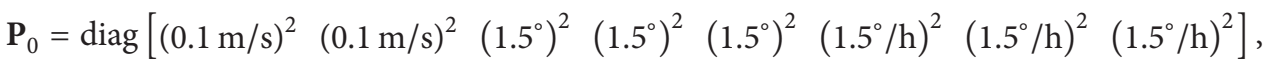

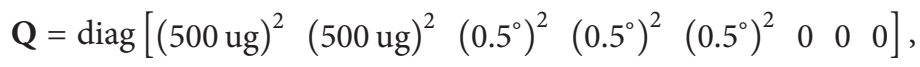

$$
\begin{aligned}
& \mathbf{R}=\operatorname{diag}\left[\begin{array}{lll}
(0.4 \mathrm{~m} / \mathrm{s})^{2} & (0.4 \mathrm{~m} / \mathrm{s})^{2} & \left.\left(0.3^{\circ}\right)^{2}\right]
\end{array}\right.
\end{aligned}
$$

Two data fusion schemes were compared. The second scheme is a transfer alignment method with the added backward-forward SINS resolution and data fusion, while the first scheme is not. In these two schemes, the same transfer alignment model introduced in Section 2 is used.

The update cycle of sensor data and navigation resolution is set as $10 \mathrm{~ms}$, and that of reference data from MINS is as 1s. As analyzed in Section 4, in every reference data cycle, navigation resolution will be executed 300 times and data fusion 3 times in scheme 2, while in scheme 1, navigation resolution is executed 100 times and data fusion only once.

In the "velocity plus yaw" matching method, accelerometer bias is unobservable, so with the sensor errors assumed in Table 1, the limit alignment accuracy of pitch and roll are $-0.4990 \mathrm{mrad}$ and $0.4990 \mathrm{mrad}$, respectively, and the limit alignment accuracy of yaw is 0 .

5.2. Simulation Results. The simulation lasts for $500 \mathrm{~s}$, and the simulation results are stored once per second. The misalignment angle curves are shown in Figure 7. The estimation of velocity error curves are in Figure 8, and the estimation curves of gyros bias in Figure 9. In Figures 7-9, the dot-dash and solid lines denote the simulation results of scheme 1 and scheme 2, respectively. The dotted lines in Figures 7 and 8 are the limit alignment accuracy of misalignment angles and velocity. And the dotted lines in Figure 9 denote the setting value of constant gyro bias.

The curves in Figure 7 show that, either in scheme 1 or scheme 2, misalignment angles can be estimated rapidly and are oscillating in small amplitudes with the swinging frequency of ship. But the tendency of misalignment curves, especially that of roll ones, indicates that estimation speed is slightly higher in scheme 2 than in scheme 1 . The statistical data about misalignment angles are shown in Table 3, and the statistical results show that the alignment accuracy of these two schemes is roughly equal after $60 \mathrm{~s}$. The curves in Figure 8 indicate that the estimation speed and accuracy for velocity error are roughly equal in scheme 1 and scheme 2 .

The curves in Figure 9 show that, in scheme 2, the estimation curves of gyro bias converge towards the setting values at about $50 \mathrm{~s}$, and the oscillating amplitudes are very small in $100 \mathrm{~s}$, while in scheme $1,200 \mathrm{~s}$ is needed for the convergence of gyro bias, and relatively large oscillations still exist even after $300 \mathrm{~s}$. The statistical mean values shown in Table 4 indicate that, in scheme 2, in the period from $50 \mathrm{~s}$ to 100 s, about $96 \%$ and $93 \%$ and $72 \%$ of gyro bias can be estimated along $x, y$, and $z$ axes, respectively. And about $99 \%, 97 \%$, and $78 \%$ can be estimated in the period from $100 \mathrm{~s}$ to $150 \mathrm{~s}$.

\section{Conclusion}

Two viewpoints are given in this paper. The first is that, in SINS, mathematical platform cuts off the direct relationship between sensor data and misalignment angles, which means that initial alignment can be fulfilled by the repeatedly resolution on a same set of sensor data. The second is that, with the added backward-forward SINS resolution and repeated data fusion on the corresponding resolution results and the external data, the alignment time can be greatly reduced. 
TABLE 3: Statistical results for misalignment angles.

\begin{tabular}{|c|c|c|c|c|c|c|}
\hline & \multicolumn{2}{|c|}{ Pitch (mrad) } & \multicolumn{2}{|c|}{ Roll (mrad) } & \multicolumn{2}{|c|}{ Yaw (mrad) } \\
\hline & Mean & Standard variance & Mean & Standard variance & Mean & Standard variance \\
\hline \multicolumn{7}{|l|}{$1 \sim 10 \mathrm{~s}$} \\
\hline Scheme 1 & -0.8225 & 1.0610 & 1.1833 & 4.9672 & -0.9375 & 1.2756 \\
\hline Scheme 2 & 0.4631 & 1.6724 & 0.2318 & 0.7429 & -0.3956 & 1.0700 \\
\hline \multicolumn{7}{|l|}{$11 \sim 20 \mathrm{~s}$} \\
\hline Scheme 1 & -0.2278 & 0.5136 & -0.0705 & 0.5156 & -1.1170 & 0.7199 \\
\hline Scheme 2 & -0.4993 & 0.5865 & 0.4806 & 0.2647 & -0.4755 & 0.8203 \\
\hline \multicolumn{7}{|l|}{$31 \sim 40 \mathrm{~s}$} \\
\hline Scheme 1 & -0.4776 & 0.4653 & 0.9794 & 0.5194 & 0.0448 & 0.9782 \\
\hline Scheme 2 & -0.6454 & 0.4553 & 0.2271 & 0.4726 & -0.0067 & 0.8820 \\
\hline \multicolumn{7}{|l|}{$41 \sim 50 \mathrm{~s}$} \\
\hline Scheme 1 & -0.8024 & 0.4560 & 1.0111 & 0.5086 & -0.3714 & 0.9385 \\
\hline Scheme 2 & -0.8563 & 0.4557 & 0.4980 & 0.6148 & -0.3609 & 0.8828 \\
\hline \multicolumn{7}{|l|}{$51 \sim 60 \mathrm{~s}$} \\
\hline Scheme 1 & -0.5430 & 0.4296 & 0.5377 & 0.4614 & -0.2183 & 0.9682 \\
\hline Scheme 2 & -0.7073 & 0.4854 & 0.5415 & 0.5531 & 0.2041 & 0.9117 \\
\hline \multicolumn{7}{|l|}{$61 \sim 500 \mathrm{~s}$} \\
\hline Scheme 1 & -0.5075 & 0.4498 & 0.4154 & 0.4852 & -0.0877 & 0.9168 \\
\hline Scheme 2 & -0.6541 & 0.4505 & 0.4815 & 0.4831 & 0.0709 & 0.9011 \\
\hline Limited accuracy & -0.4990 & & 0.4990 & & 0 & \\
\hline
\end{tabular}

TABLE 4: Statistical results for gyro bias.

\begin{tabular}{|c|c|c|c|c|c|c|}
\hline & \multicolumn{2}{|c|}{ Pitch (mrad) } & \multicolumn{2}{|c|}{ Roll (mrad) } & \multicolumn{2}{|c|}{ Yaw (mrad) } \\
\hline & Mean & Standard variance & Mean & Standard variance & Mean & Standard variance \\
\hline \multicolumn{7}{|l|}{$51 \sim 100 \mathrm{~s}$} \\
\hline Scheme 1 & -0.4139 & 0.4563 & -0.7990 & 0.9143 & 0.7591 & 0.4807 \\
\hline Scheme 2 & 0.5172 & 0.0757 & 0.5335 & 0.1329 & 0.6390 & 0.1588 \\
\hline \multicolumn{7}{|l|}{$101 \sim 150 \mathrm{~s}$} \\
\hline Scheme 1 & 0.3474 & 0.2102 & -0.3793 & 0.4709 & 1.9998 & 0.3182 \\
\hline Scheme 2 & 0.5037 & 0.0505 & 0.4847 & 0.0499 & 0.6072 & 0.0628 \\
\hline \multicolumn{7}{|l|}{$151 \sim 200 \mathrm{~s}$} \\
\hline Scheme 1 & 0.5742 & 0.0632 & 0.2499 & 0.1254 & 0.8496 & 0.2778 \\
\hline Scheme 2 & 0.4943 & 0.0382 & 0.4703 & 0.0284 & 0.5196 & 0.0256 \\
\hline \multicolumn{7}{|l|}{$201 \sim 250 \mathrm{~s}$} \\
\hline Scheme 1 & 0.4790 & 0.0283 & 0.2713 & 0.0744 & 0.5335 & 0.0648 \\
\hline Scheme 2 & 0.4961 & 0.0224 & 0.4718 & 0.0231 & 0.5255 & 0.0224 \\
\hline \multicolumn{7}{|l|}{$251 \sim 300 \mathrm{~s}$} \\
\hline Scheme 1 & 0.5189 & 0.0344 & 0.4203 & 0.0630 & 0.5292 & 0.0530 \\
\hline Scheme 2 & 0.5019 & 0.0217 & 0.4752 & 0.0258 & 0.5113 & 0.0236 \\
\hline \multicolumn{7}{|l|}{$301 \sim 500 \mathrm{~s}$} \\
\hline Scheme 1 & 0.5156 & 0.0272 & 0.4122 & 0.0410 & 0.5683 & 0.0412 \\
\hline Scheme 2 & 0.4990 & 0.0116 & 0.4823 & 0.0169 & 0.5134 & 0.0129 \\
\hline Setting value & 0.5 & & 0.5 & & 0.5 & \\
\hline
\end{tabular}

With the above two viewpoints: (1) a backward-forward SINS resolution algorithm is designed in detail, and simulation results indicate that a ship can move from the end to the origin with the backward resolution and move from the origin to the end with the forward. (2) A rapid transfer alignment algorithm is also designed in detail, in which the backward-forward resolution and two more operations for the estimation of gyro bias are added in one reference data update cycle. In addition, the correctness of its algorithm is proved by simulation. The simulation result produced with 


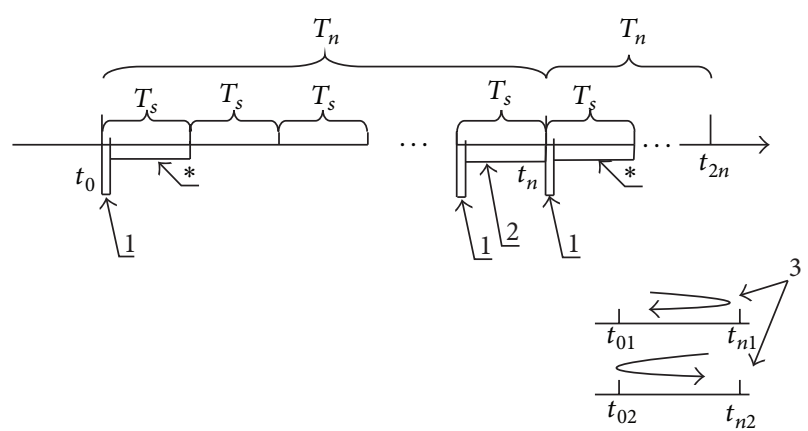

(1) Sensor data sampling, storing and navigation resolution

(2) Reference data sampling, storing and data fusion

(3) Reverse and forward calculation

* CPU idle

FIGURE 6: Calculation process for alignment program.

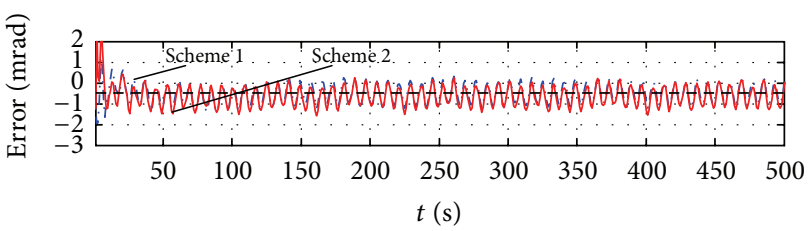

(a) Pitch

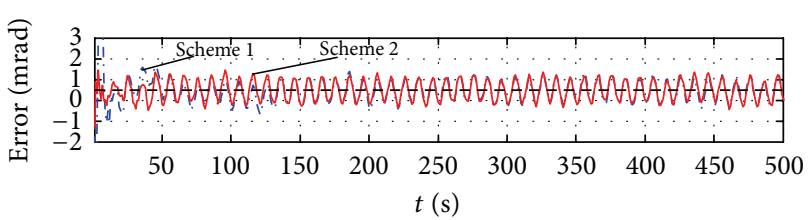

(b) Roll

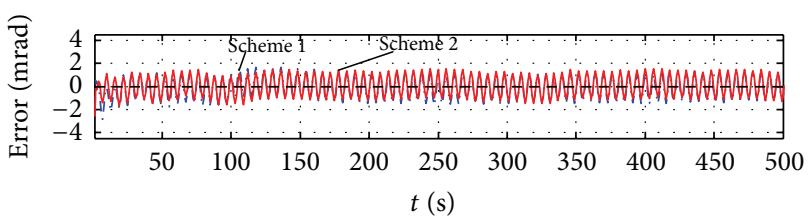

(c) Yaw

FIGURE 7: Estimation curves for misalignment angles.

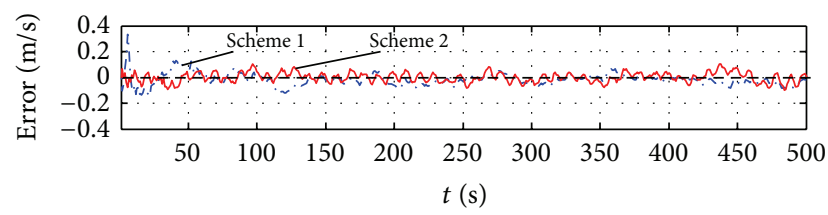

(a) East velocity

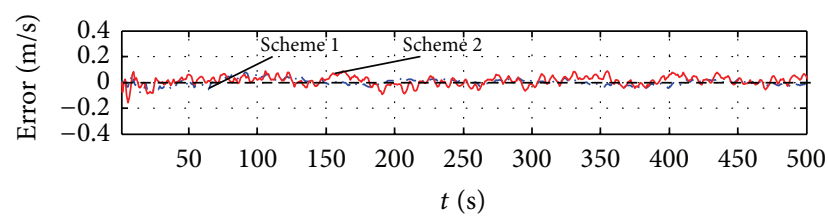

(b) North velocity

FIGURE 8: Estimation curves for velocity errors.

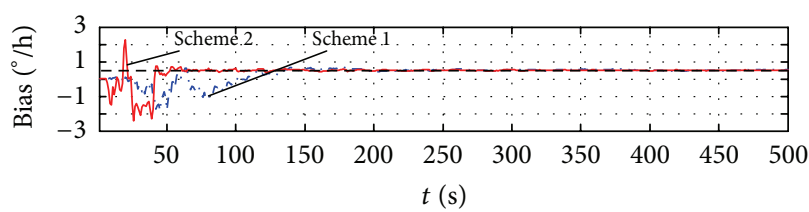

(a) $x$ gyro

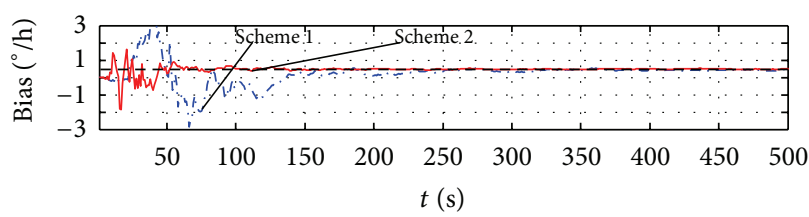

(b) $y$ gyro

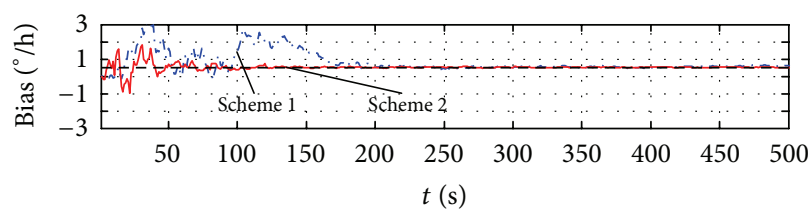

(c) $z$ gyro

FIgURE 9: Estimation curves for gyro bias.

the method in this paper indicates that the alignment time is reduced from $300 \mathrm{~s}$ to $100 \mathrm{~s}$, compared with that of the transfer alignment method without the added backwardforward SINS resolution and data fusion.

One problem, which may be encountered in engineering applications with this transfer alignment method, is studied, and a possible solution is given in which RTOS is introduced to distribute computer resources coordinately.

\section{Acknowledgments}

This work was supported in part by the National Natural Science Foundation $(61004125,61273056)$ and Chinese university research and operation expenses (104.205.2.5).

\section{References}

[1] J. Kain and J. Cloutier, "Rapid transfer alignment for tactical weapon applications," in Proceedings of the AIAA Guidance, Navigation and Control Conference, pp. 1290-1300, Boston, Mass, USA, 1989.

[2] K. Spalding, "An efficient rapid transfer alignment filter," in Proceedings of the AIAA Guidance, Navigation and Control Conference, pp. 1276-1286, Hilton Head Island, SC, USA, 1992.

[3] K. Shortelle and W. Graham, "Advanced alignment concepts for precision-guided weapons," in Proceedings of the National Technical Meeting of the Institute of Navigation, pp. 131-142, Anaheim, Calif, USA, January 1995.

[4] W. Graham and K. Shortelle, "Advanced transfer alignment for inertial navigators (A-train)," in Proceedings of the National Technical Meeting of the Institute of Navigation, pp. 113-124, Anaheim, Calif, USA, January 1995.

[5] K. J. Shortelle, W. R. Graham, and C. Rabourn, "F-16 flight tests of a rapid transfer alignment procedure," in Proceedings of the 
IEEE Position Location and Navigation Symposium, pp. 379-386, Palm Springs, Calif, USA, April 1996.

[6] P. D. Groves, "Optimising the transfer alignment of weapon INS," Journal of Navigation, vol. 56, no. 2, pp. 323-335, 2003.

[7] D. Goshen-Meskin and I. Y. Bar-Itzhack, "Observability analysis of piece-wise constant systems. II. Application to inertial navigation in-flight alignment (military applications)," IEEE Transactions on Aerospace and Electronic Systems, vol. 28, no. 4, pp. 1068-1075, 1992.

[8] X. H. Cheng, D. J. Wan, and X. Zhong, "Study on observability and its degree of strapdown inertial navigation system," Journal of Southeast University, vol. 37, no. 6, pp. 6-10, 1997.

[9] L.-H. Zhu, X.-H. Cheng, and Y.-Y. He, "Filter convergence criterion in transfer alignment," Journal of Chinese Inertial Technology, vol. 19, no. 3, pp. 277-285, 2011.

[10] S. Wang, Z. Wang, Y. Zhu, and B. Wang, "Monitoring on ship hull deformation and correction for heading and attitude information," Journal of Chinese Inertial Technology, vol. 15, no. 6, pp. 635-641, 2007.

[11] D. R. Tarrant, C. Jones, and D. Lin, "Rapid and robust transfer alignment," in Proceedings of the 1st IEEE Regional Conference on Aerospace Control Systems, pp. 758-762, 1993.

[12] J. Lyou and Y. C. Lim, "Transfer alignment considering measurement time delay and ship body flexure," Journal of Mechanical Science and Technology, vol. 23, no. 1, pp. 195-203, 2009.

[13] B.-C. Zhou, X.-H. Cheng, and D.-J. Liu, "Analysis method of observable degree based on spectral decomposition in SINS transfer alignment," Journal of Chinese Inertial Technology, vol. 18, no. 5, pp. 518-522, 2010.

[14] D. H. Titterton and J. L. Weston, Strapdown Inertial Navigation Technology, Lavenham Press Ltd., London, UK, 2nd edition, 2004.

[15] R. B. Miller, "A new strapdown attitude algorithm," Journal of Guidance, Control, and Dynamics, vol. 6, no. 4, pp. 287-291, 1983.

[16] P. G. Savage, "Strapdown inertial navigation integration algorithm design - part 1: attitude algorithms," Journal of Guidance, Control, and Dynamics, vol. 21, no. 1, pp. 19-28, 1998.

[17] P. G. Savage, "Strapdown inertial navigation integration algorithm design - part 2: velocity and position algorithms," Journal of Guidance, Control, and Dynamics, vol. 21, no. 2, pp. 208-221, 1998.

[18] P. J. Gates and N. M. Lynn, Ships, Submarines and the Sea, Brassey's, London, UK, 1990. 


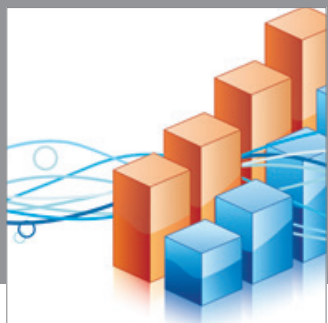

Advances in

Operations Research

mansans

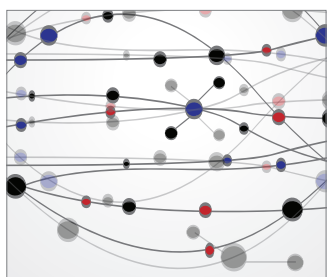

The Scientific World Journal
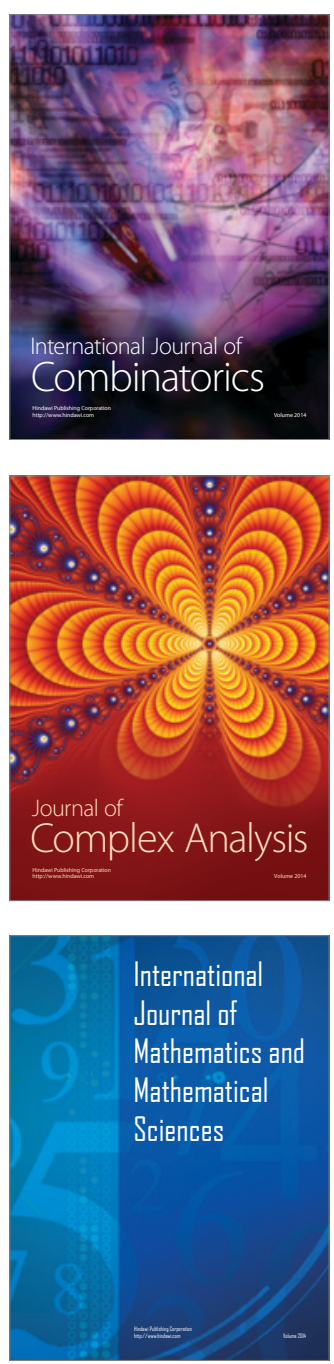
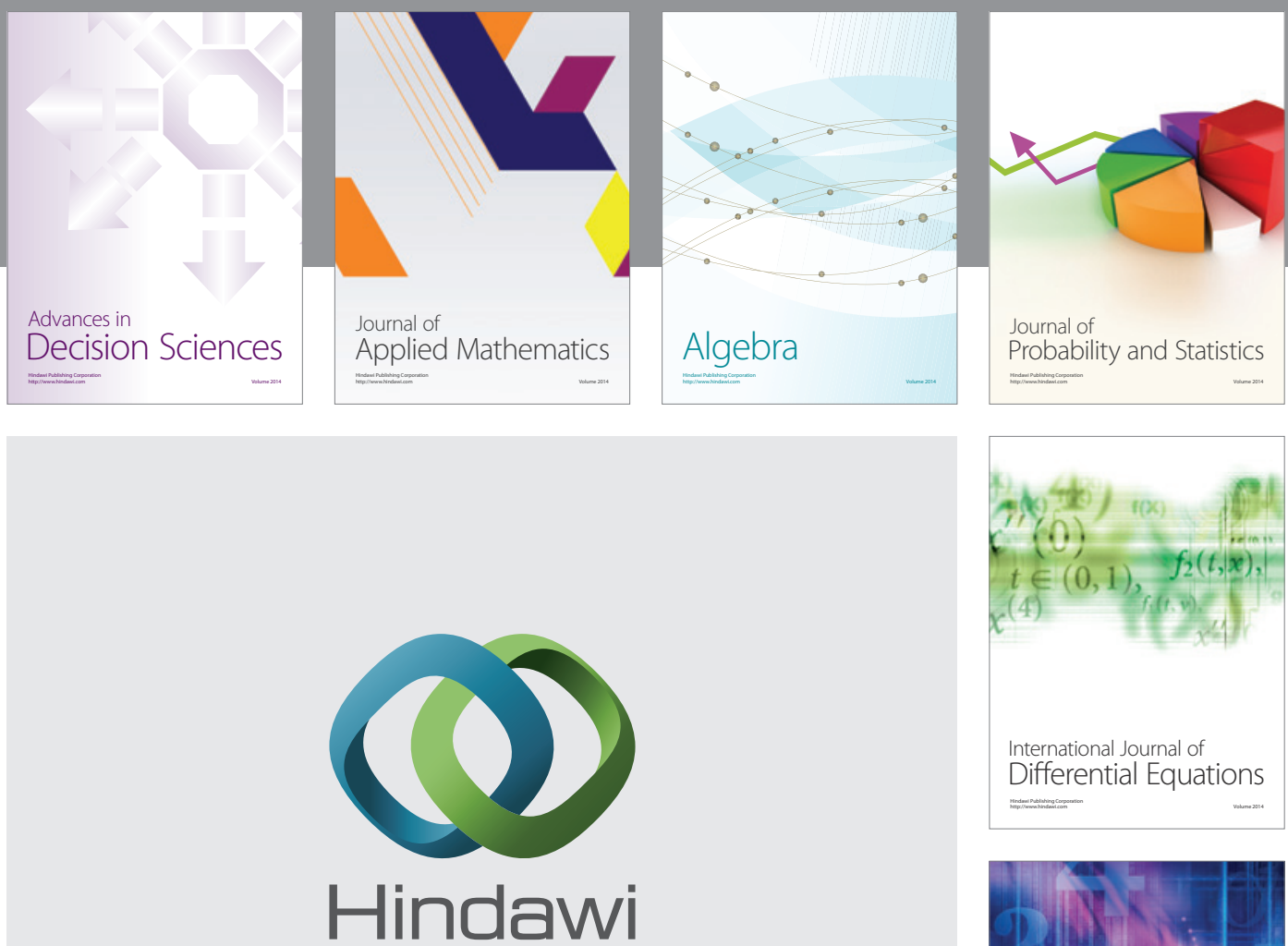

Submit your manuscripts at http://www.hindawi.com
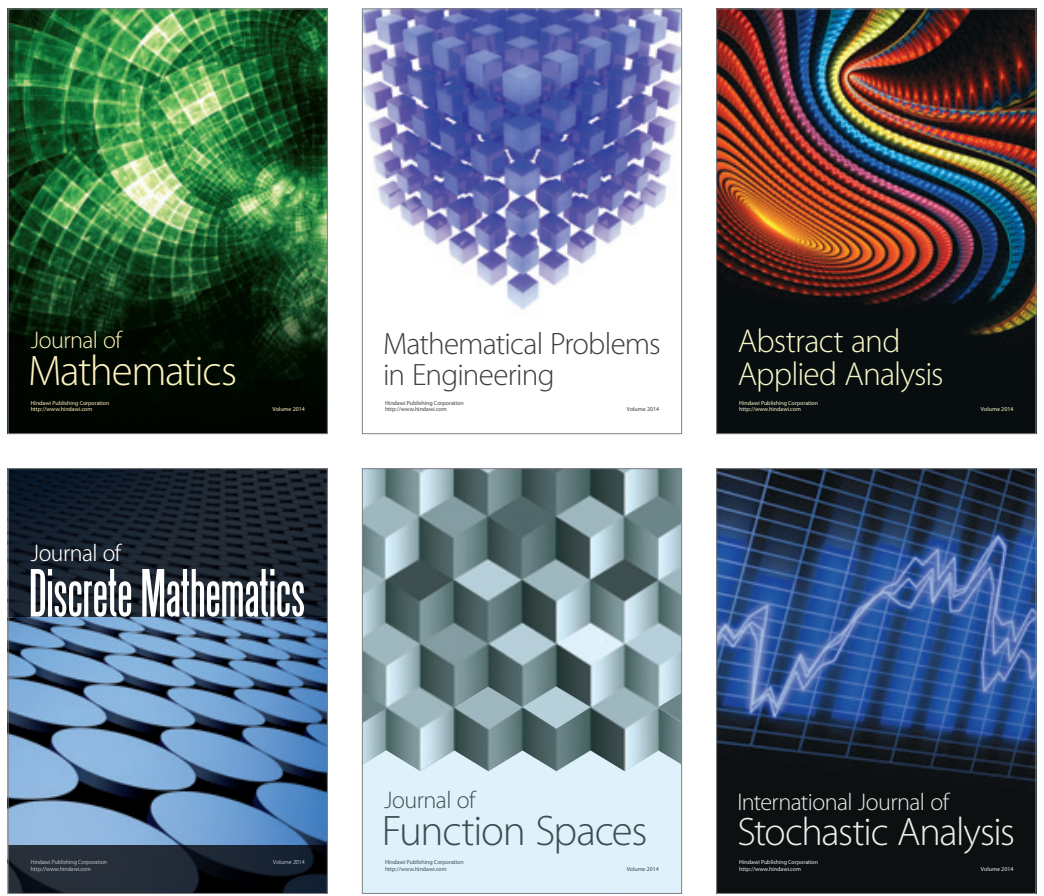

Journal of

Function Spaces

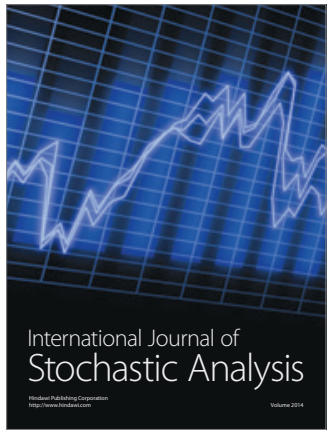

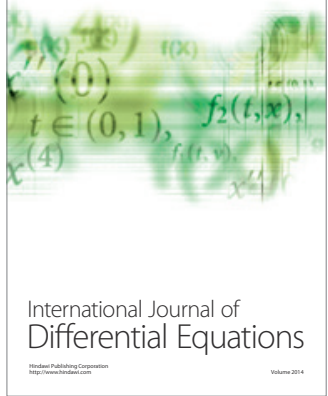
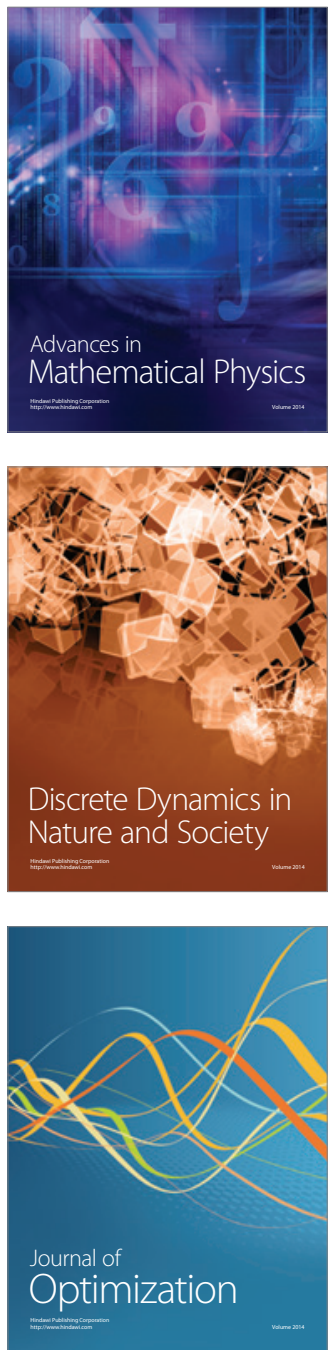